\title{
Diagnosis of Ovary-Containing Indirect Inguinal Hernia of the Canal of Nuck by Ultrasound
}

\author{
Khaleda Parvin Rekha ${ }^{1}$, Sohel Abdullah ${ }^{2}$, Mashah Binte Amin ${ }^{3}$ \\ Received: February 27, 2016 Accepted: December 11, 2016 \\ doi: http://dx.doi.org/10.3329/jemc.v7i1.30750
}

\begin{abstract}
In women, the round ligament is attached to the uterus near the origin of the fallopian tube, and a small evagination of parietal peritoneum accompanies the round ligament through the inguinal canal to the labium majorum. This small evagination of parietal peritoneum, named the canal of Nuck in women, is the equivalent of the processus vaginalis in men. Incomplete obliteration of the processus vaginalis causes indirect inguinal hernia or hydrocele of the canal of Nuck, a very rare condition in women. Here, we report a case of ovary-containing hernia of the canal of Nuck that was diagnosed with ultrasonography and was surgically confirmed. Gray scale and color Doppler ultrasonographic features studied were: the site and the size of the hernia, the texture of the hernia contents and the presence or absence of blood flow in the hernia contents.
\end{abstract}

Key words: Hydrocele; Canal of Nuck; Ultrasonography; Magnetic resonance imaging; Inguinal hernia

J Enam Med Col 2017; 7(1): 39-41

\section{Introduction}

In women, the round ligament is attached to the uterus near the origin of the fallopian tube and a small evagination of parietal peritoneum accompanies the round ligament through the inguinal canal to the labium majorum. This small evagination of parietal peritoneum, named the canal of Nuck in women, is the equivalent of the processus vaginalis in men.

In the case of a hernia of the canal of Nuck in female infants, early diagnosis is important when the hernia contains an ovary, because incarceration of the ovary is common and has been reported in up to $43 \%$ of cases. Therefore, a female infant with an inguinal hernia should be evaluated to determine whether the ovarian contents are present.

Although several cases of ovary-containing hernias of the canal of Nuck have been reported, imaging findings have rarely been described. In addition, although ultrasonography is the first line diagnostic examination for the evaluation of inguinal lesions, sonographic descriptions of ovary-containing hernias of the canal of Nuck are scant. ${ }^{1}$ Here we report this case to describe the sonographic finding of ovary-containing hernia of the canal of Nuck and its importance.

\section{Case report}

A female baby of 11 months and 29 days age was brought to Accident and Emergency Department on the day before her $1^{\text {st }}$ birthday party due to painful swelling on left groin. Physical examination revealed tender left inguinal swelling extending into labia majora and increasing in size while crying (Fig 1). Laboratory findings were unremarkable.

1. Specialist, Department of Radiology \& Imaging, United Hospital Ltd., Dhaka

2. Sonologist, Department of Radiology \& Imaging, United Hospital Ltd., Dhaka

3. Assistant Professor, Department of Radiology \& Imaging, Enam Medical College \& Hospital, Savar, Dhaka

Correspondence Khaleda Parvin Rekha, Email: parvinrekha79@gmail.com 


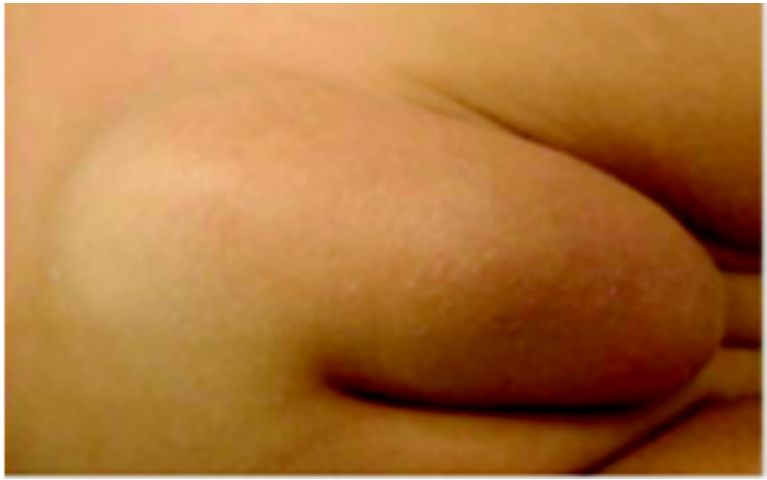

Fig 1. Swelling in left groin extending to labium majora

The baby was referred to the Department of Radiology for ultrasonography. A linear high-frequency probe (Voluson E8) was used to delineate groin structures.

Gray scale ultrasonography revealed

-Herniation of intra-abdominal contents through left inguinal canal towards labia majora (Fig 2).

-Collection of free fluid within the canal of Nuck (Fig 2).

-An ovoid structure of mixed echogenicity having peripherally-located tiny cysts representing ovary within the canal of Nuck (Fig 3).

Doppler study showed position of inferior epigastric vessel clearly medial to the neck of the hernial sac, hence the swelling was categorized as indirect inguinal hernia (Fig 4). Color Doppler and power Doppler techniques identified the presence of blood flow signals both within the mass and throughout the hernia's neck. Thus the possibility of strangulation was excluded (Fig 5).
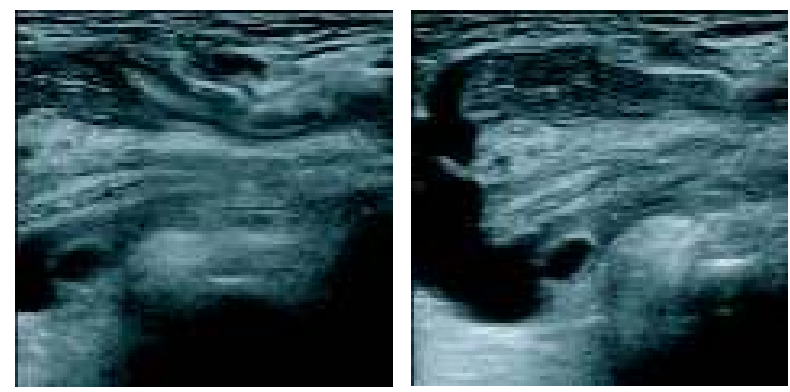

Fig 2. Sonographic images showing protrusion of intra-abdominal structures through left inguinal canal towards canal of Nuck associated with free fluid

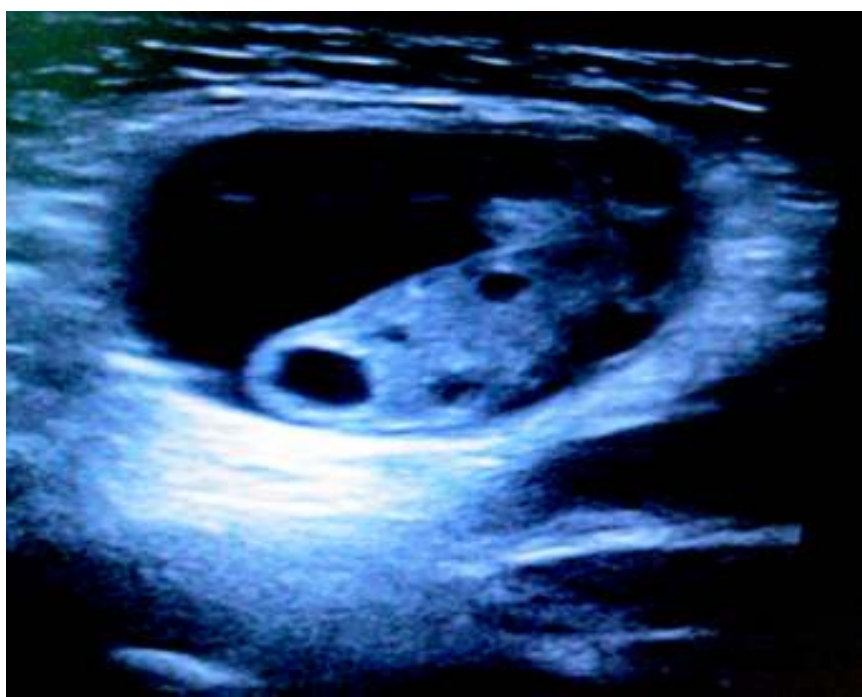

Fig 3. Ovary within the canal of Nuck having tiny peripherally-placed cysts representing follicles

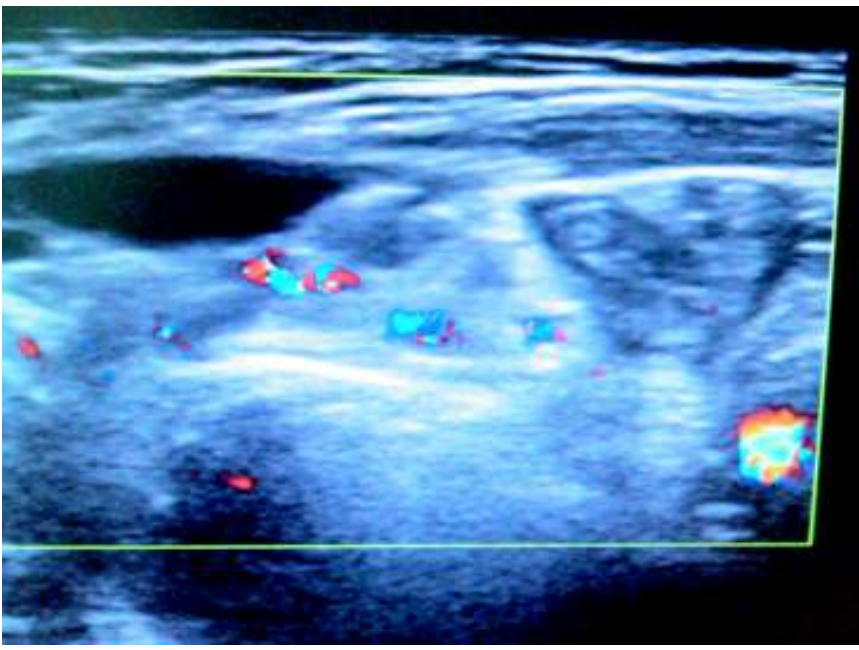

Fig 4. Doppler study showing presence of vascularity within contents of hernial sac excluding strangulation
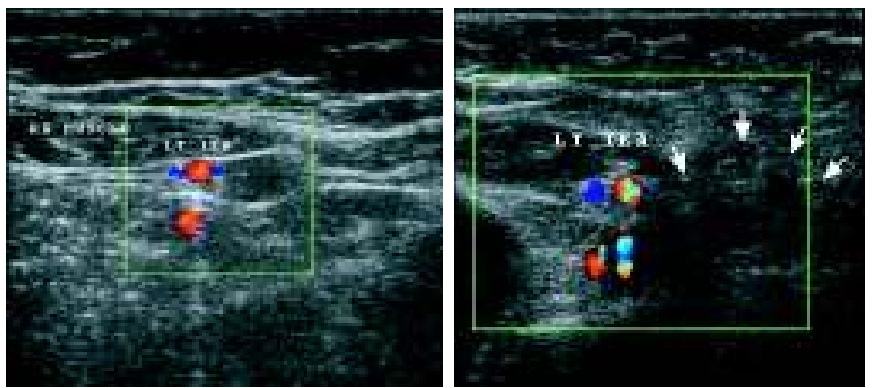

Fig 5. Doppler images of left inferior epigastric vessel showing neck of the hernial sac lateral to the origin of left inferior epigastric artery confirming that the hernia is indirect 
As the hernia was non-reducible, the patient underwent surgery which revealed presence of an indirect inguinal hernia containing the left ovary and left fallopian tube.

\section{Discussion}

The canal of Nuck was first described by Anton Nuck in $1691 .^{2}$ It is an abnormal patent pouch of parietal peritoneum extending into the labia majora of women through inguinal canal (Fig 6). It is analogous to patent processus vaginalis in male which arises during the $6^{\text {th }}$ month and is normally obliterated by the $8^{\text {th }}$ month of gestation. Incomplete obliteration of this canal can result in either an indirect inguinal hernia or a hydrocele and even endometriosis. ${ }^{1}$

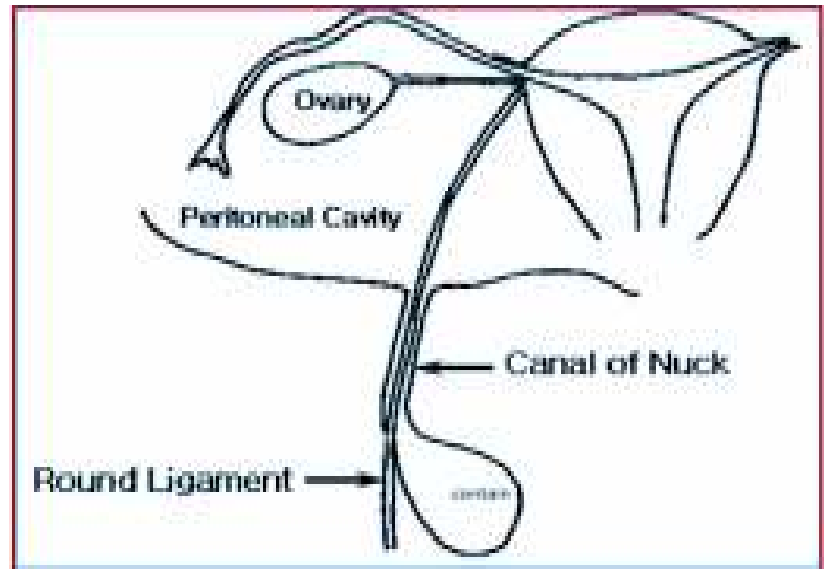

Fig 6. Illustration showing patent pouch of peritoneum extending anterior to the round ligament in relation with the uterus, fallopian tube and ovary

Inguinal hernias in children may contain omental fat, bowel or urinary bladder and in rare cases an ovary (along with the round ligament in $15 \%$ cases). The majority of ovary-containing hernias which may sometimes extend to the labia majora affect children younger than 5 years of age. ${ }^{1}$

Ovary-containing hernias that are non-reducible by manoeuvres are treated with elective surgical repair. In patients with ovarian torsion, emergency surgery should be performed in order to avoid infarction and infertility. High frequency ultrasound is the primary imaging modality of choice as it is an easy, cheap and readily available tool combining high diagnostic efficacy without using harmful radiation.
Ovary-containing hernias usually measure less than 2 $\mathrm{cm}$ in size and are usually seen with an ovoid solid mass which may be isoechoic but may contain hypoechoic cysts measuring $1--7 \mathrm{~mm}$. The cysts may be peripherally-located as they represent ovarian follicles and constitute an important ultrasonographic finding of an ovary-containing hernia. The absence of peristalsis is another finding consistent with ovary-containing hernias. Strangulated ovaries may appear enlarged and heterogeneous in echogenicity.

Color Doppler technique should always be used to assess the vascularity of the herniated ovary which can exclude suspicion of ovarian torsion. Pulsed-wave Doppler technique normally shows low-resistance arterial waveforms in the ovarian vessels. In cases of torsion, no blood flow is evident within the ovary. A worsening of the symptoms should raise suspicion of ovarian torsion.

CT should be avoided due to radiation and should be performed only when ultrasonography is not available in order to exclude ovarian torsion. An ovarian torsion will appear enlarged with decreased enhancement. Coronal reformatted images better illustrate the ovarian morphology. ${ }^{3}$

An ovary-containing hernia of the canal of Nuck is a very rare type of inguinal mass in girls. It can be diagnosed correctly almost always with point-of-care high-resolution ultrasonographic devices equipped with high-frequency probes. Even without signs of strangulation, hernias containing ovaries should be managed with early surgical reduction.

\section{References}

1. Yang DM, Kim HC, Kim SW, Lim SJ, Park SJ, Lim JW. Ultrasonographic diagnosis of ovary-containing hernias of the canal of Nuck. Ultrasonography 2014; 33(3): 178-183.

2. Anton Nuck from whonamedit.com, the dictionary of medical eponyms. Available at: http://www.whonamedit.com/ doctor.cfm/2293.html. Accessed October 2015.

3. Vasileios R, Christina U, Ioannis P, Dimitrios R. Inguinal hernia containing an ovary and fallopian tube: ultrasonographic findings correlated with surgery. Eurorad 2015. Available at: http://www.eurorad.org/case.php?id= 12536. Accessed September 2015. 